\title{
Prognostic Impact of the Tumor Marker CA 15-3 in Patients With Breast Cancer and Bone Metastases Treated With Palliative Radiotherapy
}

\author{
Carsten Nieder ${ }^{\mathrm{a}, \mathrm{b}, \mathrm{c}}$, Astrid Dalhauga, b, Ellinor Haukland ${ }^{\mathrm{a}}$, Bard Mannsaker ${ }^{\mathrm{a}}$, \\ Adam Pawinski ${ }^{\mathrm{a}}$
}

\begin{abstract}
Background: The aim of the study was to explore the prognostic impact of different abnormal blood tests and the tumor marker CA 15-3 as well as established parameters such as disease extent and receptor status in patients with bone metastases from breast cancer who received palliative radiotherapy in addition to contemporary systemic treatment.
\end{abstract}

Methods: This was a retrospective uni- and multivariate analysis of 118 female patients treated in the time period from 2007 to 2014 (median follow-up 28 months).

Results: The median age was 61 years and the median time interval from the initial diagnosis of breast cancer was 57 months (median time interval from metastatic disease to radiotherapy was 7 months). Only $16 \%$ of patients had normal serum CA 15-3. HER2 receptor status correlated with CA 15-3. The median survival was 17.6 months (lowest CA 15-3 quartile), 14.7 months (intermediate), and 6.9 months (highest quartile) $(\mathrm{P}=0.002)$. However, multivariate analysis showed that survival was influenced by extent of extra-skeletal metastases, pleural metastases/effusion, lung metastases, estrogen receptor status, serum C-reactive protein, and anemia with need for blood transfusion (all $\mathrm{P}<0.05)$ rather than CA 15-3.

Conclusions: Survival was highly variable. The tumor marker CA 15-3 did not provide independent prognostic information. Nevertheless, the results of simple blood tests contributed to the multivariate prognostic model.

Keywords: Biomarker; Bone metastases; Breast cancer; Prognostic factors; Radiotherapy

\footnotetext{
Manuscript accepted for publication July 18, 2016

${ }^{a}$ Department of Oncology and Palliative Medicine, Nordland Hospital, 8092 Bodo, Norway

bepartment of Clinical Medicine, Faculty of Health Sciences, University of Tromso, 9037 Tromso, Norway

${ }^{\mathrm{c} C}$ Corresponding Author: Carsten Nieder, Department of Oncology and Palliative Medicine, Nordland Hospital, 8092 Bodo, Norway.

Email: carsten.nieder@nlsh.no
}

doi: https://doi.org/10.14740/jocmr2653w

\section{Introduction}

Patients with metastatic breast cancer often have widespread disease, for example in the lungs, pleura, liver, lymph nodes and bones. Relatively few patients present with oligometastatic disease [1]. Use of palliative radiotherapy is common in patients with painful bone metastases, which represent a large group $[2,3]$. While the chance of long-term survival is higher in patients with limited tumor burden and less aggressive histological type, substantial individual variation exists [4]. Performance status, organ function, biological disease characteristics and eligibility for effective local and systemic treatment are important prognostic and predictive factors [5]. Prognostic models might be helpful in pre-treatment assessment [6]. In this regard, the role of the tumor marker CA 15-3 has not been studied extensively. Therefore, we evaluated the survival and prognostic factors, including a panel of blood tests, in patients treated with palliative radiotherapy for bone metastases.

\section{Methods}

\section{Patients and treatment}

This retrospective intention-to-treat study included 118 consecutive female patients with bone metastases from histologically confirmed breast cancer who received palliative external beam radiotherapy at Nordland Hospital Bodo. All patients were treated between 2007 and 2014. Some presented with synchronous bone metastases at first cancer diagnosis, others with delayed or metachronous skeletal involvement. Systemic treatment was given according to the guidelines of the Norwegian Breast Cancer Group (NBCG), which are stratified by biological subtype and available online. Radionuclide treatment was not available.

\section{Blood tests}

Lactate dehydrogenase (LDH), albumin, hemoglobin, C-reactive protein (CRP) and alkaline phosphatase (ALP) were part of routine laboratory and radiology assessment in patients with metastatic breast cancer. However, some patients had incom- 
Table 1. Patient Characteristics Before Radiotherapy (RT) and Survival Outcomes $(n=118)$

\begin{tabular}{|c|c|c|c|c|}
\hline Parameter & $\mathbf{n}$ & $\%$ & Median survival, months & P-value \\
\hline Estrogen receptor (ER) positive & 91 & 77 & 15.6 & \\
\hline Estrogen receptor (ER) negative & 27 & 23 & 5.8 & 0.004 \\
\hline HER2 positive ${ }^{\mathrm{a}}$ & 20 & 18 & 14.4 & \\
\hline HER2 negative ${ }^{a}$ & 90 & 82 & 12.4 & 0.65 \\
\hline Triple negative $^{\mathrm{a}}$ & 22 & 20 & 5.5 & \\
\hline ER positive HER2 negative ${ }^{a}$ & 68 & 62 & 15.5 & \\
\hline ER negative HER2 positive ${ }^{a}$ & 5 & 5 & 11.4 & \\
\hline ER and HER2 positive ${ }^{\mathrm{a}}$ & 15 & 14 & 15.9 & $0.007^{\mathrm{b}}$ \\
\hline Metastases at first diagnosis ${ }^{\mathrm{a}}$ & 17 & 15 & 6.9 & \\
\hline Metachronous metastatic disease ${ }^{\mathrm{a}}$ & 98 & 85 & 14.7 & 0.13 \\
\hline Interval from metastatic disease to $\mathrm{RT} \leq 1$ month $^{\mathrm{a}}$ & 32 & 28 & 17.6 & \\
\hline Interval from metastatic disease to RT $2-6$ months $^{\mathrm{a}}$ & 24 & 21 & 13.9 & \\
\hline Interval from metastatic disease to RT $7-12$ months $^{\mathrm{a}}$ & 14 & 12 & 7.5 & \\
\hline Interval from metastatic disease to RT $13-59$ months $^{\mathrm{a}}$ & 35 & 30 & 9.0 & \\
\hline Interval from metastatic disease to $\mathrm{RT} \geq 60$ months $^{\mathrm{a}}$ & 10 & 9 & 9.0 & $0.02^{b}$ \\
\hline No extra-skeletal visceral metastases & 57 & 48 & 22.9 & \\
\hline One site of visceral metastases, e.g. lung(s) & 43 & 36 & 8.2 & \\
\hline More than one site of visceral metastases & 18 & 15 & 5.1 & $0.0001^{\mathrm{b}}$ \\
\hline Liver metastases & 36 & 31 & 7.3 & 0.0001 \\
\hline Lung metastases & 38 & 32 & 7.3 & 0.004 \\
\hline Pleural metastases/effusion & 19 & 16 & 3.9 & 0.0001 \\
\hline Brain metastases & 4 & 3 & 3.4 & 0.0001 \\
\hline ECOG PS 0 & 32 & 27 & 27.3 & \\
\hline ECOG PS 1 & 38 & 32 & 15.6 & \\
\hline ECOG PS 2 & 32 & 27 & 10.3 & \\
\hline ECOG PS 3 - 4 & 16 & 14 & 3.1 & $0.0001^{\mathrm{b}}$ \\
\hline Received no previous RT & 26 & 22 & 14.9 & \\
\hline One previous course & 51 & 43 & 16.9 & \\
\hline At least two previous courses & 41 & 35 & 9.0 & $0.01^{\mathrm{b}}$ \\
\hline Serum albumin normal ${ }^{\text {a }}$ & 94 & 84 & 15.5 & \\
\hline Serum albumin low ${ }^{\mathrm{a}}$ & 18 & 16 & 5.1 & 0.001 \\
\hline Serum LDH normal ${ }^{\mathrm{a}}$ & 45 & 45 & 27.0 & \\
\hline Serum LDH high $^{\mathrm{a}}$ & 56 & 55 & 6.9 & 0.0001 \\
\hline Serum ALP normal ${ }^{\mathrm{a}}$ & 47 & 45 & 21.1 & \\
\hline Serum ALP high ${ }^{\mathrm{a}}$ & 58 & 55 & 9.0 & 0.0001 \\
\hline Serum CRP normal ${ }^{\mathrm{a}}$ & 55 & 50 & 22.9 & \\
\hline Serum CRP high $(\leq 30)^{\mathrm{a}}$ & 29 & 26 & 12.7 & \\
\hline Serum CRP high $(31-60)^{\mathrm{a}}$ & 13 & 12 & 5.8 & \\
\hline Serum CRP high $(>60)^{\mathrm{a}}$ & 14 & 13 & 5.0 & $0.0001^{\mathrm{b}}$ \\
\hline Hemoglobin normal $^{\mathrm{a}}$ & 80 & 71 & 15.9 & \\
\hline Hemoglobin low ${ }^{\mathrm{a}}$ & 33 & 29 & 8.2 & 0.004 \\
\hline Received blood transfusion before RT & 9 & 8 & 9.0 & 0.01 \\
\hline CA $15-3$ normal $^{\text {a }}$ & 14 & 16 & 36.2 & \\
\hline CA 15-3 high ${ }^{\mathrm{a}}$ & 75 & 84 & 12.2 & 0.007 \\
\hline
\end{tabular}

anformation not available in all patients. ${ }^{b}$ P-value calculated over all strata. ECOG PS: Eastern Cooperative Oncology Group performance status; LDH: lactate dehydrogenase; ALP: alkaline phosphatase; CRP: C-reactive protein. 
plete tests performed. Tumor marker analyses were performed at the discretion of the treating oncologist. The hospital's electronic patient record system was used to collect all baseline data including laboratory analyses. The latter had to be no older than 2 weeks before the first fraction of radiotherapy, mirroring our previous study [6]. Elevated LDH was defined as $\geq 205 \mathrm{U} / \mathrm{L}$ according to the hospitals' reference value (low albumin $<34 \mathrm{~g} / \mathrm{L}$; high ALP $\geq 105 \mathrm{U} / \mathrm{L}$; normal CRP $<5 \mathrm{mg} / \mathrm{L}$; low hemoglobin $<11.7 \mathrm{~g} / \mathrm{dL}$; blood transfusion was given if hemoglobin was $<10 \mathrm{~g} / \mathrm{dL})$. Normal CA 15-3 was defined as 0 - $25 \mathrm{kIE} / \mathrm{L}$.

\section{Statistical methods}

Actuarial survival from the first day of radiotherapy was calculated with the Kaplan-Meier method. Survival curves of different groups were compared with the log-rank test. Multivariate analysis consisted of Cox regression (backward conditional method). Associations between different variables of interest were assessed with the Chi-square or Fisher exact probability test (two-tailed). A P-value $\leq 0.05$ was considered statistically significant. Twenty-five patients were alive at last follow-up (June 15, 2015). Surviving patients had a median follow-up of 28 months. This study did not require approval from the Regional Committee for Medical and Health Research Ethics (REK Nord), because retrospective quality-of-care analyses are permitted without committee vote. All analyses were carried out according to our institutions' guidelines and with permission to access the patients' data.

\section{Results}

\section{Patient characteristics}

While 17 patients (15\%) had no previous history of cancer, i.e. bone metastases at initial diagnosis or de novo stage IV disease, the vast majority developed metastases at later time points. The median age of our patients was 61 years (range 33 - 87 years). The median time interval from the initial diagnosis of breast cancer was 57 months (range 1 - 384 months) and the median time interval from metastatic disease to radiotherapy was 7 months (range 1 - 180 months). The patient characteristics are shown in Table 1. The most common fractionation regimen was 10 fractions of 3 Gy $(55 \%)$ followed by 5 fractions of 4 Gy (24\%). Fifty patients (42\%) had spinal target volumes. Forty-two patients $(36 \%)$ received simultaneous radiotherapy to two target volumes and $16(14 \%)$ to at least three target volumes. All but one patient completed their prescribed course of radiotherapy.

\section{Tumor marker analyses}

CA 15-3 was analyzed in 89 patients (75\%) and normal in 14 of these $(16 \%)$. The median value was $61 \mathrm{kIE} / \mathrm{L}$ (range 9 30,000 and interquartile range $(25-75 \%) 32.5$ - 208). Patients

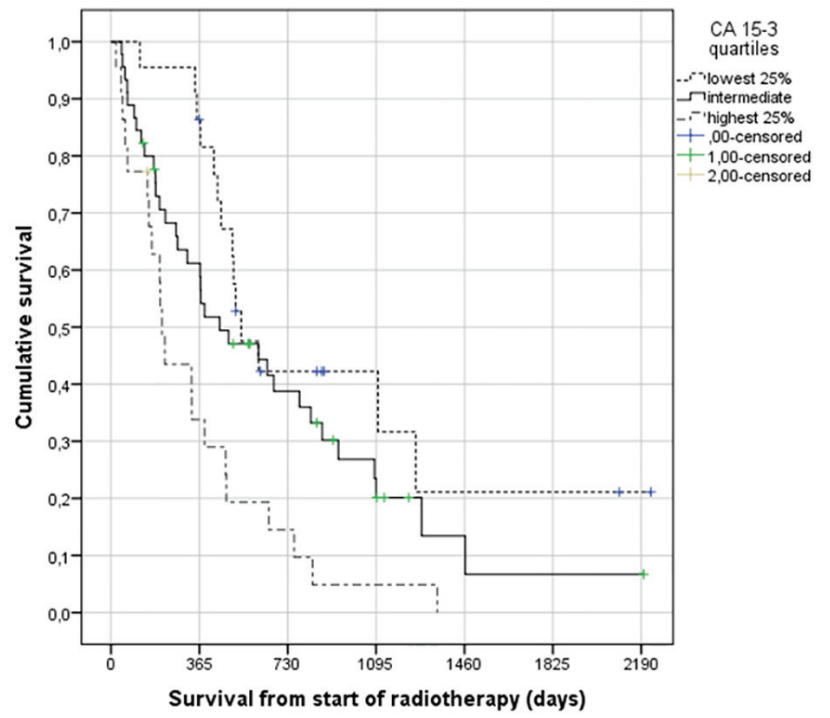

Figure 1. Actuarial Kaplan-Meier survival curves for 89 patients with bone metastases from breast cancer stratified by serum CA 15-3 level (median 17.6 for lowest quartile $(<32.5 \mathrm{kIE} / \mathrm{L}$ ) versus 14.7 months for intermediate levels and 6.9 months for highest quartile (> $208 \mathrm{kIE} / \mathrm{L}), \mathrm{P}$ $=0.002$ ), log-rank test.

with HER2 positive primary tumors had significantly higher likelihood of normal CA $15-3$ (36\% vs. 13\% in HER2 negatives, $\mathrm{P}=0.049$ ). No significant correlation between patterns of metastatic disease, extra-skeletal disease extent or estrogen receptor status and CA 15-3 abnormality was observed. Figure 1 shows the correlation between CA 15-3 and survival (median 17.6 months, 14.7 months, and 6.9 months, $\mathrm{P}=0.002$ ).

\section{Overall survival}

Median survival was 12.7 months and $22 \%$ of the patients were alive after 3 years. Median survival from initial diagnosis of metastatic disease was 20 months. As shown in Table 1, a large number of patient and disease characteristics were associated with survival after radiotherapy. These included for example breast cancer type, patterns of metastatic disease, CA 15-3, LDH, albumin, CRP, hemoglobin and ALP, but not age or radiotherapy-related factors. All parameters with significant $\mathrm{P}$-value were included in a multivariate regression analysis, which confirmed six independent prognostic factors: absence or limited extent of extra-skeletal metastases $(P=0.0001)$, no pleural metastases/effusion $(\mathrm{P}=0.0001), \mathrm{CRP}<5 \mathrm{mg} / \mathrm{L}(\mathrm{P}=$ $0.0001)$, positive estrogen receptor $(\mathrm{P}=0.001)$, no lung metastases $(\mathrm{P}=0.001)$, and no need for blood transfusion $(\mathrm{P}=$ $0.048)$.

\section{Discussion}

Bone metastases from breast cancer are a common clinical challenge for oncologists worldwide [1, 5]. Jensen et al estimated the overall and annual incidence of skeletal metastases 
and related events (SREs) in newly diagnosed breast cancer patients in Denmark from 1999 to 2007 using the Danish National Patient Registry [7]. Of the 35,912 patients with breast cancer, $178(0.5 \%)$ presented with skeletal involvement at the time of initial cancer diagnosis, and of these, $43 \%$ developed an SRE during follow-up. The term SRE relates to pathological fractures, orthopedic surgery, spinal cord compression and utilization of radiotherapy. A total of 1,272 of 35,690 (3.6\%) patients without distant disease at first diagnosis developed skeletal metastases during a median follow-up time of 3.4 years. Among these patients, $46 \%$ subsequently developed an SRE. The 5-year survival rate of patients with bone metastases was $8 \%[8]$.

Given that survival is highly variable, prognostic models are necessary to inform choice of treatment and counsel patients. As a result of previous research [6], we were interested in serum biomarkers, in particular the tumor marker CA 15-3. In previous studies that included multivariate models, elevated CA 15-3 before surgery was significantly associated with tumor size, axillary node involvement and advanced stage [9, 10]. Moreover, in stage-matched analyses prognosis of patients with elevated serum CA 15-3 was worse compared to those with normal value. Further studies demonstrated that serial postoperative surveillance of CA 15-3 values during follow-up may be useful for early detection of recurrent breast cancer [11-13].

In a retrospective study, Geng et al analyzed the association of serum CA 15-3 levels with clinicopathological parameters in women with metastatic breast cancer and determined whether the elevation of the tumor marker was correlated with metastatic sites [14]. In their study, 136 patients had only one metastatic site and 148 patients had involvement of several sites. Sixty-seven percent of patients with several sites of disease had an increased CA 15-3 level. This figure was significantly higher than the $47 \%$ reported in women with a single metastasis $(P=0.001)$. In a different study, increased CA 15-3 levels were found more frequently in patients with liver metastases and in those with pleural effusion [15]. Whereas Yerushalmi and coworkers did not identify significant differences in CA 15-3 levels between different sites of metastasis [16], Al-Jarallah et al reported that the highest proportions of pathologically elevated CA 15-3 levels were detected in women with bone metastases [17]. In our study, the only clinicopathological parameter associated with elevated CA 15-3 levels was HER2 receptor status. Our study shares limitations with previous ones, e.g. the retrospective study design. Because only 89 patients had their tumor marker analyzed, many subgroups were relatively small and therefore the statistical power was not optimal.

A typical patient in our study was a postmenopausal woman with estrogen receptor positive and HER2 negative disease who developed metachronous metastases (Table 1). According to the national guidelines, such patients receive zoledronic acid and several lines of sequential hormonal and cytotoxic therapy, depending on visceral tumor load, response to previous therapy and other parameters. We found a large number of patient and disease characteristics to be associated with survival after radiotherapy. These included for example breast cancer type (shortest survival for triple negative status, median 5.5 months), patterns of metastatic disease (longest survival for bone only disease, median 22.9 months), CA 15-3, LDH, albumin, hemoglobin, CRP and ALP, but not age. However, only six independent prognostic factors were confirmed by multivariate analysis: presence of extra-skeletal metastases together with number of involved sites, pleural metastases/effusion, lung metastases, elevated CRP, negative estrogen receptor, and anemia with need for blood transfusion. Interestingly, performance status was less important than patterns of metastases and the other factors just mentioned. A potential explanation might be that poor performance status resulting from bone pain often improves rapidly after radiotherapy. The impact of hormone receptor status is well known from the literature $[1,4,5]$. Just like LDH, ALP and albumin, increased CA 15-3 was not significant in our multivariate analysis.

In contrast, Turanli et al found CA 15-3 to be an independent factor associated with overall survival [18]. However, their study included a different patient population with isolated bone metastases treated between June 2004 and January 2007 (129 consecutive female patients). Serum CA 15-3 levels were elevated in $63 \%$ of patients with isolated bone metastases. In multivariate analysis, the serum CA 15-3 level influenced survival with a relative risk of 2.5 (95\% confidence interval: 1.36 - 4.64, $\mathrm{P}=0.003)$. In an older study of a more diverse patient population (1997 - 2001; $\mathrm{n}=492)$, which was better comparable to ours, James et al also reported that CA 15-3 was associated with survival [5]. These authors studied patients with newly diagnosed metastatic breast cancer, including those with metachronous presentation and those with metastatic disease at the time of initial diagnosis of breast cancer. Several of the factors analyzed were significantly associated with better survival in women with bone metastases. These were estrogen receptor status $(\mathrm{P}=0.0003)$, histological grade $(\mathrm{P}=0.034)$, additional sites of metastatic disease $(\mathrm{P}=0.0004)$, patient age $(\mathrm{P}=0.0003)$, number of hot spots on isotope bone scan $(\mathrm{P}=$ $0.040)$ and metastasis-free interval $(\mathrm{P}=0.0045)$. In addition, the tumor markers CA 15-3 and CEA were significantly related to survival too $(\mathrm{P}=0.0026$ and 0.017 , respectively). In multivariate analysis, long metastasis-free interval, absence of metastases at sites other than bone, estrogen receptor positivity, and normal CEA and CA 15-3 values at presentation contributed independently towards longer survival from time of presentation with skeletal metastases.

Bidard et al analyzed CA 15-3 levels in 1,298 patients, of whom $892(69 \%)$ had increased values [19]. According to the univariate analysis, abnormal CA 15-3 was significantly associated with decreased overall survival (hazard ratio: 1.59, 95\% confidence interval: $1.31-1.93, \mathrm{P}<0.0001)$. When added to their clinicopathological models, logistic regression tests showed that the dichotomized marker ( $<$ upper limit of normal vs. $\geq$ upper limit of normal) added some prognostic information but increases in $\mathrm{C}$-indices were negligible. Their main focus was on circulating tumor cells, which contributed better prognostic information than CA 15-3. Other innovative markers are also of considerable interest [20-23]. However, none of these are part of routine clinical practice yet. A drawback of most studies, resulting in difficulties with comparison of the results, is that not all potential prognostic factors were included, e.g. CRP and hemoglobin. 


\section{Conclusions}

Many patients survived for several years rather than a few months only. The tumor marker CA 15-3 did not provide independent prognostic information. Nevertheless, the results of simple blood tests contributed to the multivariate model.

\section{Disclosures}

The authors declare that they have no conflicts of interest. No funding was received.

\section{References}

1. Giuliani J, Bonetti A. Trends in survival for patients with metastatic breast cancer: is survival improving? Tumori. 2015;101(4):347-352.

2. Feyer PC, Steingraeber M. Radiotherapy of Bone Metastasis in Breast Cancer Patients - Current Approaches. Breast Care (Basel). 2012;7(2):108-112.

3. Valeriani M, Scaringi C, Blasi L, Carnevale A, De Sanctis $\mathrm{V}$, Bonome $\mathrm{P}$, Bracci S, et al. Multifraction radiotherapy for palliation of painful bone metastases: 20 Gy versus 30 Gy. Tumori. 2015;101(3):318-322.

4. Steinauer K, Gross MW, Huang DJ, Eppenberger-Castori S, Guth U. Radiotherapy in patients with distant metastatic breast cancer. Radiat Oncol. 2014;9:126.

5. James JJ, Evans AJ, Pinder SE, Gutteridge E, Cheung KL, Chan S, Robertson JF. Bone metastases from breast carcinoma: histopathological - radiological correlations and prognostic features. Br J Cancer. 2003;89(4):660-665.

6. Nieder C, Dalhaug A, Haukland E, Mannsaker B, Pawinski A. Tumor marker analyses in patients with brain metastases: patterns of practice and implications for survival prediction research. Tumour Biol. 2015;36(8):64716476.

7. Jensen AO, Jacobsen JB, Norgaard M, Yong M, Fryzek JP, Sorensen HT. Incidence of bone metastases and skeletal-related events in breast cancer patients: a population-based cohort study in Denmark. BMC Cancer. 2011;11:29.

8. Yong M, Jensen AO, Jacobsen JB, Norgaard M, Fryzek JP, Sorensen HT. Survival in breast cancer patients with bone metastases and skeletal-related events: a populationbased cohort study in Denmark (1999-2007). Breast Cancer Res Treat. 2011;129(2):495-503.

9. Park BW, Oh JW, Kim JH, Park SH, Kim KS, Lee KS. Preoperative CA 15-3 and CEA serum levels as predictor for breast cancer outcomes. Ann Oncol. 2008;19(4):675681.

10. Lee JS, Park S, Park JM, Cho JH, Kim SI, Park BW. Elevated levels of preoperative CA 15-3 and CEA serum levels have independently poor prognostic significance in breast cancer. Ann Oncol. 2013;24(5):1225-1231.
11. Nicolini A, Tartarelli G, Carpi A, Metelli MR, Ferrari $\mathrm{P}$, Anselmi L, Conte $\mathrm{M}$, et al. Intensive post-operative follow-up of breast cancer patients with tumour markers: CEA, TPA or CA15.3 vs MCA and MCA-CA15.3 vs CEA-TPA-CA15.3 panel in the early detection of distant metastases. BMC Cancer. 2006;6:269.

12. Pedersen AC, Sorensen PD, Jacobsen EH, Madsen JS, Brandslund I. Sensitivity of CA 15-3, CEA and serum HER2 in the early detection of recurrence of breast cancer. Clin Chem Lab Med. 2013;51(7):1511-1519.

13. Di Gioia D, Heinemann V, Nagel D, Untch M, Kahlert $\mathrm{S}$, Bauerfeind I, Koehnke T, et al. Kinetics of CEA and CA15-3 correlate with treatment response in patients undergoing chemotherapy for metastatic breast cancer (MBC). Tumour Biol. 2011;32(4):777-785.

14. Geng B, Liang MM, Ye XB, Zhao WY. Association of CA 15-3 and CEA with clinicopathological parameters in patients with metastatic breast cancer. Mol Clin Oncol. 2015;3(1):232-236.

15. Tampellini M, Berruti A, Gorzegno G, Bitossi R, Bottini A, Durando A, De Matteis A, et al. Independent factors predict supranormal CA 15-3 serum levels in advanced breast cancer patients at first disease relapse. Tumour Biol. 2001;22(6):367-373.

16. Yerushalmi R, Tyldesley S, Kennecke H, Speers C, Woods R, Knight B, Gelmon KA. Tumor markers in metastatic breast cancer subtypes: frequency of elevation and correlation with outcome. Ann Oncol. 2012;23(2):338-345.

17. al-Jarallah MA, Behbehani AE, el-Nass SA, Temim L, Ebraheem AK, Ali MA, Szymendera JJ. Serum CA-15.3 and CEA patterns in postsurgical follow-up, and in monitoring clinical course of metastatic cancer in patients with breast carcinoma. Eur J Surg Oncol. 1993;19(1):74-79.

18. Turanli S, Cetin A. Prognostic role of serum cancer antigen 15-3 in breast cancer patients with isolated bone metastases. Biomarkers. 2010;15(5):418-423.

19. Bidard FC, Peeters DJ, Fehm T, Nole F, Gisbert-Criado $\mathrm{R}$, Mavroudis D, Grisanti S, et al. Clinical validity of circulating tumour cells in patients with metastatic breast cancer: a pooled analysis of individual patient data. Lancet Oncol. 2014;15(4):406-414.

20. Hu XY, Huang XY, Ma J, Zuo Y, Luo NB, Lai SL, Su DK. GSTT1 and GSTM1 polymorphisms predict treatment outcome for breast cancer: a systematic review and meta-analysis. Tumour Biol. 2016;37(1):151-162.

21. Lei B, Xu SP, Liang XS, Li YW, Zhang JF, Zhang GQ, Pang D. Long non-coding RNA MVIH is associated with poor prognosis and malignant biological behavior in breast cancer. Tumour Biol. 2016;37(4):5257-5264.

22. Ravelli A, Reuben JM, Lanza F, Anfossi S, Cappelletti MR, Zanotti L, Gobbi A, et al. Breast cancer circulating biomarkers: advantages, drawbacks, and new insights. Tumour Biol. 2015;36(9):6653-6665.

23. Lin S, Gan Z, Han K, Yao Y, Min D. Interleukin-6 as a prognostic marker for breast cancer: a meta-analysis. Tumori. 2015;101(5):535-541. 